PROCEEDINGS OF THE

AMERICAN MATHEMATICAL SOCIETY

Volume 132, Number 8, Pages 2185-2195

S 0002-9939(04)07065-0

Article electronically published on March 24, 2004

\title{
QUASI-MINIMAL ABELIAN GROUPS
}

\author{
B. GOLDSMITH, S. ÓhÓGÁIN, AND S. WALLUTIS
}

(Communicated by Stephen D. Smith)

\begin{abstract}
An abelian group $G$ is said to be quasi-minimal (purely quasiminimal, directly quasi-minimal) if it is isomorphic to all its subgroups (pure subgroups, direct summands, respectively) of the same cardinality as $G$. Obviously quasi-minimality implies pure quasi-minimality which in turn implies direct quasi-minimality, but we show that neither converse implication holds. We obtain a complete characterisation of quasi-minimal groups. In the purely quasi-minimal case, assuming GCH, a complete characterisation is also established. An independence result is proved for directly quasi-minimal groups.
\end{abstract}

\section{$\S 1$. INTRODUCTION}

The concept of quasi-minimality was first introduced in a topological context (see e.g. 6], [11 and [12]). Given a collection $\mathcal{C}$ of topological spaces a quasi-order, i.e., a reflexive and transitive but not necessarily anti-symmetric relation, "sub", is defined on $\mathcal{C}$ by: $Y$ sub $X$ if $Y$ is homeomorphic to a subspace of $X$, for $X, Y \in \mathcal{C}$. Then a space $X \in \mathcal{C}$ is said to be quasi-minimal if $Y$ sub $X$ and $|Y|=|X|$ implies $X$ is homeomorphic to $Y$. The present work investigates collections of abelian groups from a similar standpoint.

An abelian group $G$ is said to be quasi-minimal (purely quasi-minimal, directly quasi-minimal, respectively) if $G$ is isomorphic to each of its subgroups (pure subgroups, direct summands, respectively) of the same cardinality as $G$. It is possible to completely characterise quasi-minimal groups. Large classes of purely quasiminimal groups can also be characterised; however, to achieve a complete characterisation we have assumed the general continuum hypothesis $(\mathrm{GCH})$. It is not clear to us at this stage whether it is necessary to make such an additional set-theoretic assumption. We note that in the directly quasi-minimal case an example can be given to show that the direct quasi-minimality of a group may be undecidable in ZFC.

Our notation is standard and largely in accord with Fuchs [4] and [5], which contains all undefined group-theoretic terms used herein; an exception is that we write mappings on the right and write $A \sqsubset B$ to denote that $A$ is a direct summand of $B$. The necessary set-theoretic background for this work is contained in [3]. In this paper the term "group" shall always denote an abelian group.

Received by the editors April 17, 2002 and, in revised form, September 25, 2002.

2000 Mathematics Subject Classification. Primary 20 K99.

(C)2004 American Mathematical Society 


\section{§. QUASI-MINIMAL GROUPS}

Since a finite group is trivially quasi-minimal we concentrate on groups of cardinality $\kappa$ where $\kappa$ is a fixed but arbitrary infinite cardinal. Let $A b_{\kappa}$ denote the set of groups of cardinality $\kappa$. As mentioned above, a group $G \in A b_{\kappa}$ is quasi-minimal if $G$ is isomorphic to all its subgroups of cardinality $\kappa$. Since any group $G$ can be written in the form $G=D \oplus R$ where $D$ is divisible and $R$ is reduced, we immediately get that a quasi-minimal group is either divisible or reduced. The following lemma gives a further reduction.

Lemma 2.1. If $G$ is quasi-minimal, then $G$ is either torsion-free or a p-group.

Proof. If $|t G|=|G|$, then $G \cong t G$, since $G$ is quasi-minimal. Now, $t G=\bigoplus_{p \in \Pi} G_{p}$ where $G_{p}$ is a $p$-group for all $p$. Choose $p$ such that $G_{p} \neq 0$ and then $t G=$ $G_{p} \oplus \bigoplus_{q \neq p} G_{q}$, so $|G|=\left|G_{p}\right|$ or $|G|=\left|\bigoplus_{q \neq p} G_{q}\right|$. If $|G|=\left|\bigoplus_{q \neq p} G_{q}\right|$, then $G \cong \bigoplus_{q \neq p} G_{q}$, a contradiction, since $G_{p} \neq 0$. Therefore $|G|=\left|G_{p}\right|$ and so $G \cong G_{p}$ is a $p$-group. Now suppose $|t G|<|G|$. Then $|G / t G|=|G|=\kappa$. If $\kappa=\aleph_{0}$, then $t G$ is finite and so $t G \sqsubset G$, since $t G$ is pure in $G$. Then $G=t G \oplus C$ where $C$ is torsionfree and $|G|=|C|=\aleph_{0}$, and so $G \cong C$ (and $t G=0$ ), i.e. $G$ is torsion-free. If $\kappa>\aleph_{0}$, then $r(G / t G)=|G / t G|$; so we can choose $\kappa$ linearly independent elements $\bar{g}_{\alpha}=g_{\alpha}+t G, \alpha<\kappa$. Let $C=\left\langle g_{\alpha}: \alpha<\kappa\right\rangle \leq G$. The group $C$ is torsion-free since if $c=\sum_{\alpha<\kappa} k_{\alpha} g_{\alpha} \in t G$ where $k_{\alpha}=0$ for almost all $\alpha$, then $\sum_{\alpha<\kappa} k_{\alpha} g_{\alpha}+t G=t G$, so $\sum_{\alpha<\kappa} k_{\alpha} \bar{g}_{\alpha}=0$ and hence $k_{\alpha}=0$ for all $\alpha$, i.e. $c=0$. Now $|G|=|C|$ and so $G \cong C$, i.e. $G$ is torsion-free.

Quasi-minimal torsion-free groups and quasi-minimal p-groups are easily characterised.

Proposition 2.2. If $G$ is torsion-free of cardinality $\kappa$, then $G$ is quasi-minimal if and only if $G$ is free, of rank $\kappa$ if $\kappa>\aleph_{0}$, and of rank 1 if $\kappa=\aleph_{0}$.

Proof. Firstly, $\mathbb{Z}$ is quasi-minimal since the only non-zero subgroups of $\mathbb{Z}$ are of the form $n \mathbb{Z} \cong \mathbb{Z}$ where $n \in \mathbb{N}$. Also $\bigoplus_{\kappa} \mathbb{Z}$ is quasi-minimal for $\kappa>\aleph_{0}$ since if $H \leq \bigoplus_{\kappa} \mathbb{Z} \quad\left(\kappa>\aleph_{0}\right)$ with $|H|=\kappa$, then $H$ is free of rank $\kappa$ and so $H \cong G$. It remains to show that these are the only torsion-free quasi-minimal groups.

If $\kappa=\aleph_{0}$, let $0 \neq g \in G$ and consider $\langle g\rangle$. Since $G$ is torsion-free $|\langle g\rangle|=\aleph_{0}$ and so $G \cong\langle g\rangle \cong \mathbb{Z}$.

Now consider $\kappa>\aleph_{0}$. In this case $r(G)=\kappa$ so $G$ has $\kappa$ linearly independent elements $\left\{g_{\alpha}: \alpha<\kappa\right\}$. Now $\left\langle g_{\alpha}: \alpha<\kappa\right\rangle=\bigoplus_{\alpha<\kappa}\left\langle g_{\alpha}\right\rangle$ and $\left|\bigoplus_{\alpha<\kappa}\left\langle g_{\alpha}\right\rangle\right|=\kappa$. Therefore $G \cong \bigoplus_{\alpha<\kappa}\left\langle g_{\alpha}\right\rangle \cong \bigoplus_{\kappa} \mathbb{Z}$

Proposition 2.3. If $G$ is a p-group of cardinality $\kappa \geq \aleph_{0}$, then $G$ is quasi-minimal if and only if $G \cong \mathbb{Z}\left(p^{\infty}\right)$ or $G \cong \bigoplus_{\kappa} \mathbb{Z}(p)$ for some prime $p$.

Proof. Firstly, $\mathbb{Z}\left(p^{\infty}\right)$ is obviously quasi-minimal since its only subgroup of cardinality $\aleph_{0}$ is itself. Also $\bigoplus_{\kappa} \mathbb{Z}(p)$ is quasi-minimal since any subgroup is of the form $\bigoplus_{\lambda} \mathbb{Z}(p)$ and if the cardinalities are equal, then $\lambda=\kappa$. So consider a quasi-minimal 
$p$-group $G$ of cardinality $\kappa \geq \aleph_{0}$. Let $B$ be a basic subgroup of $G, B=\bigoplus_{n \in \mathbb{N}} \bigoplus_{i \in I_{n}}\left\langle x_{i}\right\rangle$ with $o\left(x_{i}\right)=p^{n}$ for $i \in I_{n}$ where $I_{n}=\emptyset$ is allowed.

We first suppose $|G|>\aleph_{0}$. In this case we claim that $|G|=|G[p]|$ where $G[p]$ is the $p$-socle of $G$. If $|B|=|G|$, then $|B[p]|=\left|\bigoplus_{n \in \mathbb{N}} \underset{I_{n}}{\bigoplus}\left\langle p^{n-1} x_{i}\right\rangle\right|=\kappa$ and hence $|G[p]| \geq|B[p]|=\kappa=|G|$. If $|B|<|G|$, then $|G / B|=|G|$ and $G / B$ is divisible, so $G / B=\bigoplus_{j \in J} \mathbb{Z}\left(p^{\infty}\right)$ where $|J|=\kappa$. There exists $C \leq G$, containing $B$, such that $C / B=\bigoplus_{j \in J} \mathbb{Z}(p)$. Then $C \cong B \oplus \bigoplus_{j \in J} \mathbb{Z}(p)$, since $B$ is pure in $C$, and so $C[p]=B[p] \oplus \bigoplus_{j \in J} \mathbb{Z}(p)$. Therefore $|G|=|G / B|=\kappa=|C[p]| \leq|G[p]|$. Thus, in both cases, $|G[p]|=|G|$. Hence $G \cong G[p] \cong \bigoplus \mathbb{Z}(p)$.

If $\kappa=\aleph_{0}$, then either $|B|=|G|$ or $|B|$ is finite. If $|B|=|G|$, then we get $G \cong G[p] \cong \bigoplus_{\aleph_{0}} \mathbb{Z}(p)$, as above. On the other hand, if $B$ is finite, then $B \sqsubset G, G=$ $B \oplus D$, say, with $D \cong G / B$, divisible and infinite. Therefore $G \cong D=\bigoplus_{I} \mathbb{Z}\left(p^{\infty}\right)$ for some index set $I$. If $|I| \geq 2$, then $G$ contains a subgroup $H \cong \mathbb{Z}(p) \oplus \bigoplus_{J} \mathbb{Z}\left(p^{\infty}\right)$ where $I=J \cup\left\{i_{0}\right\}$, say. Now, $|H|=|G|$ so $G \cong H$, a contradiction, since $H$ is not divisible. Thus $|I|=1$ and $G \cong \mathbb{Z}\left(p^{\infty}\right)$.

We summarise our characterisation of quasi-minimal groups in the following theorem:

Theorem 2.4. A group $G$ of cardinality $\kappa$ is quasi-minimal if and only if

(i) $\kappa<\aleph_{0}$; or

(ii) $\left(\kappa=\aleph_{0}\right) \quad G=\mathbb{Z}, \quad \mathbb{Z}\left(p^{\infty}\right)$ or $\bigoplus_{\aleph_{0}} \mathbb{Z}(p)$; or

(iii) $\left(\kappa>\aleph_{0}\right) \quad G=\bigoplus_{\kappa} \mathbb{Z}$ or $\bigoplus_{\kappa} \mathbb{Z}(p)$.

\section{§3. PURely QUaSi-Minimal Groups}

Next we consider the purely quasi-minimal groups in $A b_{\kappa}$. Recall that a group $G \in A b_{\kappa}$ is purely quasi-minimal if $G$ is isomorphic to all its pure subgroups of cardinality $\kappa$. It is easily seen that we get the same reduction to either divisible or reduced groups as for the quasi-minimal groups. The divisible case is taken care of in the following lemma.

Lemma 3.1. For a divisible purely quasi-minimal group $G$ of cardinality $\kappa$ one of the following is true:

(i) $G \cong \mathbb{Q} \quad\left(\kappa=\aleph_{0}\right)$,

(ii) $G \cong \mathbb{Z}\left(p^{\infty}\right)$, for some $p \quad\left(\kappa=\aleph_{0}\right)$,

(iii) $G \cong \bigoplus \mathbb{Q} \quad\left(\kappa>\aleph_{0}\right)$,

(iv) $G \cong \bigoplus_{\kappa}^{\kappa} \mathbb{Z}\left(p^{\infty}\right)$, for some $p \quad\left(\kappa>\aleph_{0}\right)$.

Proof. We first note that $\mathbb{Q}, \mathbb{Z}\left(p^{\infty}\right), \bigoplus Q, \oplus \mathbb{Z}\left(p^{\infty}\right)$ are indeed purely quasi-minimal since a pure subgroup of a divisible group is again divisible.

Let $G$ be divisible and purely quasi-minimal. Then $G=\underset{I}{\bigoplus} \oplus \underset{p \in \Pi}{\bigoplus} \underset{I_{p}}{\bigoplus} \mathbb{Z}\left(p^{\infty}\right)$, for some index sets $I, I_{p}$. If $|G|=\aleph_{0}$, then it is immediate that $G$ must be isomorphic 
to either $\mathbb{Q}$ or $\mathbb{Z}\left(p^{\infty}\right)$, for some $p$. Now suppose $|G|=\kappa>\aleph_{0}$. Then either $|I|=\kappa$ or $\left|\bigoplus_{p \in \Pi} \underset{I_{p}}{\bigoplus} \mathbb{Z}\left(p^{\infty}\right)\right|=\kappa$. If $|I|=\kappa$ we have $G \cong \bigoplus_{\kappa} \mathbb{Q}$. On the other hand, if $|I|<\kappa$, then $\kappa=\sup _{p}\left|I_{p}\right|$. If $\left|I_{p}\right|=\kappa$ for some $p$, then $G \cong \bigoplus_{I_{p}} \mathbb{Z}\left(p^{\infty}\right)$. If $\left|I_{p}\right|<\kappa$ for all $p$, then there exist infinitely many $I_{p} \neq \emptyset$. Consider $H=\underset{I_{p}(p \neq q)}{\bigoplus} \mathbb{Z}\left(p^{\infty}\right)$ where $I_{q} \neq \emptyset$. Then $|H|=|G|$, so $H \cong G$, a contradiction, since $H$ has no $q$-component. We conclude that if $|I|<\kappa$, then $G \cong \underset{I_{p}}{\bigoplus} \mathbb{Z}\left(p^{\infty}\right)$ for some $p$.

Turning to the reduced case we have a further reduction to homocyclic or torsionfree groups:

Theorem 3.2. If a reduced group $G$ is purely quasi-minimal, then $G$ is either a homocyclic p-group, i.e. $G=\bigoplus_{I} \mathbb{Z}\left(p^{n}\right)$ for some index set $I$ and some $n \in \mathbb{N}$, or $G$ is torsion-free.

Proof. To begin we show that a homocyclic group is purely quasi-minimal. Let $G=\bigoplus_{i<\kappa} y_{i} \mathbb{Z}\left(p^{n}\right)$ for some $n$ and some infinite cardinal $\kappa$, and let $H \leq_{*} G$ with $|H|=|G|$. The group $H$ is again a direct sum of cyclics, $H=\bigoplus_{I}\left\langle x_{i}\right\rangle$, say, with $|I|=\kappa$ since $|H|=\kappa$. Suppose $o\left(x_{i}\right)=p^{m}$ for some $m<n$ and some $i \in I$. We have $x_{i}=\sum_{j<\kappa} k_{j} y_{j}$ where $k_{j}=0$ for almost all $j$. Since $p^{m} x_{i}=0$ it follows that $0=\sum_{j<\kappa} p^{m} k_{j} y_{j}$ and so $p^{m} k_{j} y_{j}=0$ for all $j$. Therefore $p^{n}$ divides $p^{m} k_{j}$ for all $j$, so $p$ divides $k_{j}$ for all $j$ and hence $x_{i}=p \sum_{j<\kappa} r_{j} y_{j}$ where $k_{j}=p r_{j}$ for all $j$. We get that $p$ divides $x_{i}$ in $G$ and so $p$ divides $x_{i}$ in $H$ since $H$ is pure in $G$, a contradiction, $x_{i}$ being a generator of $\left\langle x_{i}\right\rangle$ in $H$. We conclude that $o\left(x_{i}\right)=p^{n}$ for all $i \in I$ and so $H \cong G$.

Now let $G$ be a reduced purely quasi-minimal group. If $t G=0$, then $G$ is torsionfree, so suppose $t G \neq 0$. Choose $p$ such that $G_{p} \neq 0$ and $G_{p}$ is not $p$-divisible; such exists since $G$ is reduced and $t G \neq 0$. Let $B$ be a $p$-basic subgroup of $G$; clearly $B \neq 0$. We claim that $B$ cannot be torsion-free. Since $G_{p}$ is not divisible there exists some $x \in G_{p}[p]$ with finite $p$-height (see [4, 20(c)]), and as $G / B$ is $p$-divisible we have $x=p^{n} g_{n}+b_{n}$, for each $n$, where $g_{n} \in G$ and $b_{n} \in B$. Now, $p x=0$ implies $p^{n+1} g_{n}+p b_{n}=0$, so $p^{n+1} g_{n}=-p b_{n} \in p^{n+1} G \cap B=p^{n+1} B$ and hence $p b_{n}=p^{n+1} b_{n}^{\prime}$ where $b_{n}^{\prime} \in B$. If $B$ is torsion-free, then we get $b_{n}=p^{n} b_{n}^{\prime}$ and so $x=p^{n}\left(g_{n}+b_{n}^{\prime}\right)$, i.e., $p^{n}$ divides $x$ for all $n$, a contradiction.

Therefore $B=B_{0} \oplus \bigoplus_{n=1}^{\infty} B_{n}$ where $B_{0}$ is free or trivial and not all $B_{n}=0$. Let $k>0$ be the smallest integer with $B_{k} \neq 0$. Then $G=B_{k} \oplus\left(B^{*}+p^{k} G\right)$ where $B^{*}=B_{0} \oplus \bigoplus_{n k} B_{n}$. If $|G|=\left|B_{k}\right|$, then $G \cong B_{k}$ and so $G \cong \bigoplus_{\kappa} \mathbb{Z}\left(p^{k}\right)$ and hence $G$ is homocyclic. Otherwise $|G|=\left|B^{*}+p^{k} G\right|$ and hence $G \cong B^{*}+p^{k} G=H$, say. We claim that $B^{*}$ is a $p$-basic subgroup of $H$.

By definition, $B^{*}$ is a direct sum of cyclics. Also $B^{*}$ is pure in $B$ which is pure in $G$ and so $B^{*}$ is pure in $G$ and hence in $H$. Finally $B^{*}=B \cap H$ since if $b \in B \cap H$, then $b=b^{*}+p^{k} g$ where $b^{*} \in B^{*}$ and $g \in G$, and so $b-b^{*} \in p^{k} G \cap B=p^{k} B \leq B^{*}$, 
which means that $B \cap H \leq B^{*}$. The converse inclusion is obvious. Therefore $H / B^{*}=H /(B \cap H) \cong(H+B) / B=G / B$ is $p$-divisible.

Since $H \cong G$ we get $B^{*} \cong B$, a contradiction, since $B_{k} \neq 0$. Hence $|G|=\left|B_{k}\right|$ and $G$ is a homocyclic group.

It remains to consider the reduced torsion-free case. Before the characterisation can be established we need some general results on reduced torsion-free groups. First recall the definition of high subgroups.

Definition 3.3. Let $A$ be a subgroup of a torsion-free group $G$. A subgroup $K$ of $G$ is an $A$-high subgroup of $G$ if $A \cap K=0$, and if $K^{\prime} \supseteq K$ such that $A \cap K^{\prime}=0$, then $K^{\prime}=K$, i.e. $K$ is maximal with respect to the property $A \cap K=0$.

The following result is well known but we include the simple proof for completeness.

Lemma 3.4. Let $A$ be a subgroup of a torsion-free group $G$ and let $K$ be an A-high subgroup of $G$. Then $K \leq_{*} G$ and $G /(A \oplus K)$ is torsion.

Proof. Suppose that $m g \in K$ for some $g \in G \backslash K$ and some $m \in \mathbb{Z}$. Then $\langle K, g\rangle \cap A \neq$ 0 , so there exists some non-zero $c \in A$ with $c=k+n g$ for some $k \in K$ and $n \in \mathbb{Z}$. Therefore $m c=m k+m n g \in A \cap K=0$ and so $c=0$, since $A$ is torsion-free, a contradiction. Hence $m g \notin K$ if $g \notin K$ and so $K \leq_{*} G$.

For the second part consider $g \in G \backslash A \oplus K$. Then $g \in G \backslash K$ and hence $\langle K, g\rangle \cap A \neq 0$. Therefore $k+n g=c$ for some $k \in K, c \in A$ and $n \in \mathbb{Z}$. We have $n g=c-k$ and so $n(g+A \oplus K)=0$ and hence $G /(A \oplus K)$ is torsion.

Lemma 3.5. If $G$ is a reduced torsion-free purely quasi-minimal group, then $G$ is $t$-homogeneous for some type $t$.

Proof. Let $t \in T(G)$, the typeset of $G$. Then there exists some $g^{\prime} \neq 0$ in $G$ such that $t\left(g^{\prime}\right)=t$. Consider $G(t)=\{g \in G: t(g) \geq t\} \neq 0$. It is well known that $G(t) \leq_{*} G$ and we claim that $|G(t)|=|G|$.

Let $K$ be a $G(t)$-high subgroup of $G$. Lemma 3.4 now tells us that $K \leq_{*} G$ and $G /(K \oplus G(t))$ is torsion. Therefore $G=(K \oplus G(t))_{*}$ and hence $|G|=|K \oplus G(t)| \cdot \aleph_{0}=$ $|K| \cdot|G(t)| \cdot \aleph_{0}=|K|$ or $|G(t)|$. If $|G|=|K|$, then $G \cong K$, since $K \leq_{*} G$, and we get $G(t) \cong K(t) \leq G(t) \cap K=0$, a contradiction, since $G(t) \neq 0$. Therefore $|G|=|G(t)|$ and hence $G \cong G(t)$ whenever $G(t) \neq 0$. Now, if $t, s \in T(G)$, then there exist $a, b \in G$ such that $t(a)=t$ and $t(b)=s$ where the types are with respect to $G$. Since $G \cong G(t)$ and $G \cong G(s)$ we get $G(t) \stackrel{\unrhd}{\cong} G(s)$. Now, $t(a)=t(a \phi)$ and $t(b)=t\left(b \phi^{-1}\right)$ imply that $t=s$ and therefore $G$ is $t$-homogeneous.

Following Griffith [7] we make the following definition:

Definition 3.6. A linearly independent subset $S$ of a torsion-free group $G$ is quasipure independent if $\bigoplus_{x \in S}\langle x\rangle_{*}$ is a pure subgroup of $G$ where $\langle x\rangle_{*}=\langle x\rangle$ whenever $\langle x\rangle_{*}$ is cyclic.

Note that every torsion-free group has quasi-pure independent subsets and Zorn's Lemma implies that any quasi-pure independent set is contained in a maximal one.

Next we state some results concerning quasi-pure independent subsets of a torsion-free group $G$. 
Lemma 3.7. Let $G$ be any torsion-free group. Then

(i) If $T, S$ are two infinite maximal quasi-pure independent sets of $G$, then $|T|=|S|$.

(ii) If $S$ a maximal quasi-pure independent subset of $G$, then $|G| \leq(|S|+1)^{\aleph_{0}}$.

Proof. See [7, Corollary 125 and Theorem 126].

Definition 3.8. A subgroup $H$ of a torsion-free group $G$ is pure essential in $G$ if $H \leq_{*} G$ and if $A \leq G$ with $A \cap H=0$ and $A \oplus H \leq_{*} G$, then $A=0$, in other words, $G /(A \oplus H)$ is not torsion-free for any such non-zero $A \leq G$.

The following result, which we shall exploit to obtain our characterisation of purely quasi-minimal groups, is due to Griffith.

Theorem 3.9. Every torsion-free group $G$ has a completely decomposable pure essential subgroup $C$ such that $|G| \leq|C|^{\aleph_{0}}$.

Proof. See [7, Theorem 129].

We are now ready to establish the characterisation of reduced torsion-free purely quasi-minimal groups under the assumption of the Generalised Continuum Hypothesis (GCH). Recall that GCH states that the successor of any infinite cardinal $\kappa$ is $2^{\kappa}$. Note that we do not need GCH in the countable case.

Theorem $3.10(\mathrm{GCH})$. A torsion-free reduced group $G$ of cardinality $\kappa$ is purely quasi-minimal if and only if either $G \cong R\left(\kappa=\aleph_{0}\right)$ or $G \cong \bigoplus_{\kappa} R\left(\kappa>\aleph_{0}\right)$, for some rank 1 group $R$.

Proof. Firstly, we show that $R$ and $\bigoplus_{\kappa} R$ are purely quasi-minimal. If $0 \neq H \leq_{*} R$, then $R / H$ is torsion-free, which is impossible since $R$ has rank 1 . Hence the only non-zero pure subgroup of $R$ is $R$ itself and so $R$ is purely quasi-minimal. If $H \leq_{*} \bigoplus R$, then $H$ is also homogeneous completely decomposable of the same type as ${ }^{\kappa} R$. Therefore $H=\bigoplus_{I} R$ and if $|H|=\kappa>\aleph_{0}$, then $|I|=\kappa$ and so $H \cong \bigoplus R$.

Now let $G$ be a torsion-free reduced purely quasi-minimal group. Lemma 3.5 tells us that $G$ is $t$-homogeneous for some type $t$. By Theorem 3.9 there exists a pure essential completely decomposable subgroup $C$ of $G$ such that $|G| \leq|C|^{\aleph_{0}}$. Let $C=\bigoplus_{I} R$ where $R$ is a rank 1 group whose type must be $t$ since $R \leq_{*} C \leq_{*} G$.

If $|G|=\aleph_{0}$, then $|R|=|C|=|G|=\aleph_{0}$ and hence $G \cong R$. So consider $|G|=\kappa>\aleph_{0}$. If $|C|=|G|=\kappa$, then $G \cong C$ and we are finished. We wish to prove that $|C|<|G|$ is impossible. Let us assume that $|C|<|G|$ to obtain a contradiction. First note that $|C|<|G|$ implies $2^{|C|} \leq|G|$, assuming GCH, and $|G| \leq|C|^{\aleph_{0}} \leq\left(2^{|C|}\right)^{\aleph_{0}}=2^{|C|}$, so $|G|=2^{|C|}$. Now consider the short exact sequence $0 \longrightarrow C \stackrel{i}{\longrightarrow} G \stackrel{\pi}{\longrightarrow} G / C \longrightarrow 0$ where $i$ is inclusion and $\pi$ is canonical projection. The induced sequence $0 \longrightarrow \operatorname{Hom}(G / C, G) \longrightarrow \operatorname{Hom}(G, G) \longrightarrow \operatorname{Hom}(C, G)$ is exact. We claim that $\operatorname{Hom}(G / C, G)=0$.

Let $g+C \in G / C$. Then $t_{G / C}(g+C) \geq t_{G}(g)=t$ since homomorphisms do not decrease types. If $t_{G / C}(g+C)=t$, then $R \cong\langle g+C\rangle_{*}$. Denote $\langle g+C\rangle_{*}$ by $B / C$, a pure subgroup of $G / C$. Then $C \leq_{*} B$ and $B / C$ is homogeneous completely 
decomposable of type $t$ and every element of $B \backslash C$ is of type $t$ since $B \leq_{*} G$, so $C \sqsubset B$. Therefore $B=C \oplus R_{1}$ where $R_{1} \cong R$, and $B \leq_{*} G$, but this contradicts the fact that $C$ is pure essential in $G$. We conclude that $t_{G / C}(g+C)>t$ for all $g \in G$. Hence $\operatorname{Hom}(G / C, G)=0$, again since homomorphisms do not decrease types. Therefore $0 \longrightarrow \operatorname{Hom}(G, G) \longrightarrow \operatorname{Hom}(C, G)$ is exact, so $\operatorname{Hom}(G, G)$ is isomorphic to a subgroup of $\operatorname{Hom}(C, G)$ and hence $|\operatorname{Hom}(G, G)| \leq|\operatorname{Hom}(C, G)| \leq$ $|G|^{|C|}=\left(2^{|C|}\right)^{|C|}=2^{|C|}=|G|$. But $|G|>\aleph_{0}$ means that $r(G)=|G|$ and so there exists a maximal linearly independent set $X$ in $G$ of cardinality $\kappa$. Then this set $X$ contains $2^{\kappa}$ different linearly independent subsets $\{S\}$ of $G$ of cardinality $\kappa$ (see [9, p. 43]). Each of these subsets $S$ generates a pure subgroup $\langle S\rangle_{*}$ of $G$. Furthermore, if $S_{1} \neq S_{2}$, then $\left\langle S_{1}\right\rangle_{*} \neq\left\langle S_{2}\right\rangle_{*}$ since otherwise, for any $s \in S_{1} \backslash S_{2}$, we have $s \in\left\langle S_{2}\right\rangle_{*}$ and so there exist non-zero integers $n, n_{1}, \ldots, n_{k}$, for some $k$, such that $n s=n_{1} x_{1}+\cdots+n_{k} x_{k}$ with $x_{1}, \ldots, x_{k} \in S_{2}$; but this contradicts the fact that $S_{1} \cup S_{2}$ is contained in the linearly independent subset $X$ of $G$.

Now, if $K_{1}$ and $K_{2}$ are two such pure subgroups of $G$, then $G \cong K_{1}$ and $G \cong$ $K_{2}$, since $G$ is purely quasi-minimal. If $\phi_{1}: G \longrightarrow K_{1}$ and $\phi_{2}: G \longrightarrow K_{2}$ are isomorphisms, then $K_{1} \neq K_{2}$ implies $\phi_{1} \neq \phi_{2}$ and thus there exist at least $2^{\kappa}$ different endomorphisms of $G$. Therefore $2^{|G|} \leq|\operatorname{End}(G)| \leq|G|$ which is obviously a contradiction. Hence we can deduce that $|C|<|G|$ is impossible and so $|C|=|G|$ and $G$ is homogeneous completely decomposable.

Note that in Theorem 3.10, if $|G| \leq \aleph_{\omega}$, then it is enough to assume the continuum hypothesis $(\mathrm{CH})$, i.e. $2^{\aleph_{0}}=\aleph_{1}$, as the following argument shows. The Hausdorff Formula (see [8] Theorem 1.6.12]) tells us that $\aleph_{\alpha+1}^{\aleph_{\beta}}=\aleph_{\alpha+1} \cdot \aleph_{\alpha}^{\aleph_{\beta}}$ for all $\alpha, \beta \in$ Ord with $\beta<\alpha+1$; so $\aleph_{1}^{\aleph_{0}}=\aleph_{1} \cdot \aleph_{0}^{\aleph_{0}}=\aleph_{1} \cdot 2^{\aleph_{0}}=\aleph_{1}$, using CH, and a simple induction argument now gives $\aleph_{n}^{\aleph_{0}}=\aleph_{n}$ for all $n>1$. Therefore, in Theorem 3.10, if:

(i) $|G|=\aleph_{1}$ and $|C|=\aleph_{0}$, then $\mathrm{CH}$ gives us that $|G|=2^{|C|}$ and we get a contradiction as in the proof of the theorem;

(ii) $|G|=\aleph_{\alpha}$ where $1<\alpha \leq \omega$ and $|C|=\aleph_{n}, n<\alpha$, then $|C|^{\aleph_{0}}=\aleph_{n}^{\aleph_{0}}=\aleph_{n}$ $(\mathrm{CH})<\aleph_{\alpha}=|G|$, a contradiction to $|G| \leq|C|^{\aleph_{0}}$.

As in the quasi-minimal case, we summarise what we have established concerning the purely quasi-minimal groups in a theorem:

Theorem 3.11. A group $G \in A b_{\kappa}$ is purely quasi-minimal if and only if

(i) $\kappa<\aleph_{0}$ : or

(ii) $\left(\kappa=\aleph_{0}\right) \quad G=R, \quad \mathbb{Z}\left(p^{\infty}\right) \quad$ or $\bigoplus_{\aleph_{0}} \mathbb{Z}\left(p^{k}\right)$; or

(iii) $\left(\kappa>\aleph_{0}\right) \quad G=\bigoplus_{\kappa} R(G C H), \quad \bigoplus_{\kappa} \mathbb{Z}\left(p^{\infty}\right) \quad$ or $\bigoplus_{\kappa} \mathbb{Z}\left(p^{k}\right)$, where $R$ is a rank 1 group, $p$ is any prime and $k$ is any positive integer.

Note. The authors are not convinced that an additional set-theoretic hypothesis is necessary in Theorem 3.10 but see no obvious way of avoiding the use of GCH. Even the assumption that the type $R$ involved is of idempotent type does not give any significant simplification since, as observed by Lutz Strüngman, a homogeneous torsion-free group $G$ of type $R$ is purely quasi-minimal if and only if its subgroup $G_{R}=\left\{g \in G: \chi_{G}(g) \geq \chi_{R}(1)\right\}$ is purely quasi-minimal. This latter group is, of course, homogeneous of idempotent type. 


\section{§4. Directly quasi-minimal Groups}

The final type of quasi-minimal group $G$ we consider is where we require that $G$ be isomorphic only to all its direct summands of the same cardinality as itself. In this case $G$ is called directly quasi-minimal. Again the usual reduction to either divisible or reduced groups is true. We can characterise the divisible directly quasiminimal groups as in Lemma 3.1.

Lemma 4.1. For a divisible directly quasi-minimal group $G$ one of the following is true:

(i) $G \cong \mathbb{Q} \quad\left(\kappa=\aleph_{0}\right)$,

(ii) $G \cong \mathbb{Z}\left(p^{\infty}\right)$, for some $p \quad\left(\kappa=\aleph_{0}\right)$,

(iii) $G \cong \bigoplus \mathbb{Q} \quad\left(\kappa>\aleph_{0}\right)$,

(iv) $G \cong \bigoplus_{\kappa}^{\kappa} \mathbb{Z}\left(p^{\infty}\right)$, for some $p \quad\left(\kappa>\aleph_{0}\right)$.

Proof. The arguments are similar to those in the purely quasi-minimal case.

Theorem 3.2 is also true in the directly quasi-minimal case.

Theorem 4.2. If a reduced group $G$ is directly quasi-minimal, then $G$ is either a homocyclic p-group or $G$ is torsion-free.

Proof. Apply the same arguments as before.

It remains to consider the torsion-free reduced case. Every indecomposable torsion-free reduced group is trivially directly quasi-minimal. Such groups exist in abundance: Shelah [13] has shown that, for each infinite cardinal $\kappa$, there exist $2^{\kappa}$ non-isomorphic indecomposable groups of cardinality $\kappa$. Recall that a decomposable group $G$ is superdecomposable if $G$ has no indecomposable direct summands. The existence of countable superdecomposable groups was first established by Corner [1]. For a countable decomposable directly quasi-minimal group we have the following lemma.

Lemma 4.3. If $G$ is a countable torsion-free directly quasi-minimal group, then either $G$ is indecomposable or

(i) $G \cong \bigoplus_{n} G$, for all $n \in \mathbb{N}$ and so $G$ must have infinite rank;

(ii) $G^{*}=\operatorname{Hom}(G, \mathbb{Z})=0$;

(iii) $G$ is superdecomposable.

Proof. (i) Assuming $G$ is decomposable we have $G=A \oplus B$ where $|A|=|B|=$ $|G|=\aleph_{0}$. Therefore $G \cong A$ and $G \cong B$ and so $G \cong G \oplus G$. A straightforward induction now completes the proof.

(ii) Again we assume $G$ is decomposable and note that Stein's Theorem (see 4, Corollary 19.3]) tells us that $G=N \oplus F$ where $F$ is free and $N$ has no free quotient groups (or equivalently, $N^{*}=0$ ). If $F \neq 0$, then $G \cong F$ (and $N=0$ ), so $G=\mathbb{Z}$ since $G$ is directly quasi-minimal, a contradiction to the decomposability of $G$. Therefore $F=0$ and $G \cong N$ and hence $G^{*}=0$.

(iii) If $A$ is a direct summand of $G$, then, as in (i), $G \cong A$ and so $A$ is decomposable.

Note that (iii) implies (ii) in Lemma 4.3, since if $\phi$ is a non-zero homomorphism from $G$ to $\mathbb{Z}$, then $G / \operatorname{Ker} \phi \cong \operatorname{Im} \phi \cong \mathbb{Z}$ and so $G \cong \operatorname{Ker} \phi \oplus \mathbb{Z}$. 
Properties (i) and (ii) of Lemma 4.3 are not sufficient to characterise the countable decomposable directly quasi-minimal torsion-free groups as the following example shows:

Corner [2] (see also [5, Theorem 91.6]) has given an example of a countable group $G$ with countable endomorphism ring where $G \cong \bigoplus_{n} G$ for all $n$ but $G \nsubseteq \bigoplus_{\aleph_{0}} G$, and it is a standard exercise to extend this to show the existence of a family of $2^{\aleph_{0}}$ groups $G_{j}$ with $G_{j} \cong \bigoplus_{n} G_{j}$ for all $j$ but $\operatorname{Hom}\left(G_{j}, G_{i}\right)=0$ if $j \neq i$. Stein's Theorem tells us that for each ${ }^{n} j, G_{j}=N_{j} \oplus F_{j}$ where $N_{j}$ and $F_{j}$ are as in Lemma 4.3. Therefore $G_{j}^{*} \cong N_{j}^{*} \oplus F_{j}^{*}=F_{j}^{*}$. Since $G_{j}^{*} \cong G_{j}^{*} \oplus G_{j}^{*}$ we get that $F_{j}^{*} \cong F_{j}^{*} \oplus F_{j}^{*}$ and so either $F_{j}^{*}=0$ or $F_{j}^{*}$ has infinite rank. Hence the same must be true for $F_{j}$, since $F_{j}$ is free. Now, if $F_{j}$ has infinite rank, then $\left|F_{j}^{*}\right|=2^{\aleph_{0}}$ and so $\left|G_{j}^{*}\right|=2^{\aleph_{0}}$. But $G_{j}^{*} \leq \operatorname{End}\left(G_{j}\right)$, since $G_{j}$ is torsion-free, so $G_{j}^{*}$ is countable. We conclude that $F_{j}^{*}=G_{j}^{*}=0$. Now, if we take two such groups $G_{1} \neq G_{2}$, then $G_{1} \oplus G_{2}$ clearly satisfies properties (i) and (ii) but is obviously not directly quasi-minimal.

In a similar way we can show that superdecomposability is not sufficient for direct quasi-minimality:

Corner [1] (see also [5, Theorem 91.5]) has given another example of a countable superdecomposable group with the property that every non-zero idempotent $\varepsilon$ of the group has a corresponding non-zero idempotent $\zeta$ such that $\zeta=\zeta \varepsilon=\varepsilon \zeta \neq \varepsilon$. Now consider two such groups $A$ and $B$ with $\operatorname{Hom}(A, B)=\operatorname{Hom}(B, A)=0$ and set $G=A \oplus B$. We show that $G$ is superdecomposable. First of all $E(G) \cong$ $\left(\begin{array}{cc}E(A) & 0 \\ 0 & E(B)\end{array}\right)$ where $E(G), E(A), E(B)$ denote the endomorphism rings of $G, A$ and $B$, respectively. So, if $X \neq 0$ is some summand of $G$ and $\varepsilon$ is projection onto $X$, along some complementary summand, then $\varepsilon$ is an idempotent in $E(G)$ and so $\varepsilon=\left(\begin{array}{cc}\varepsilon_{a} & 0 \\ 0 & \varepsilon_{b}\end{array}\right)$, for some idempotents $\varepsilon_{a} \in E(A)$ and $\varepsilon_{b} \in E(B)$. Now there exist idempotents $\zeta_{a} \in E(A)$ and $\zeta_{b} \in E(B)$ such that $\zeta_{a}=\zeta_{a} \varepsilon_{a}=\varepsilon_{a} \zeta_{a} \neq \varepsilon_{a}$ and $\zeta_{b}=\zeta_{b} \varepsilon_{b}=\varepsilon_{b} \zeta_{b} \neq \varepsilon_{b}$. Then, setting $\zeta=\left(\begin{array}{cc}\zeta_{a} & 0 \\ 0 & \zeta_{b}\end{array}\right)$, we get that $\zeta=\zeta \varepsilon=\varepsilon \zeta \neq \varepsilon$. We have $G \zeta=G \zeta \varepsilon \subseteq G \varepsilon=X$ and $G \zeta$ is a non-zero summand of $G$, so $G \zeta$ is a non-zero summand of $X$. In fact, it can easily be verified that $X=G \zeta \oplus G(\varepsilon-\zeta)$. Therefore $G$ is a countable superdecomposable group but $G$ is obviously not directly quasi-minimal.

It is an open question whether there exist groups satisfying (i) and (iii) which are not directly quasi-minimal.

Turning to the uncountable case, every purely quasi-minimal group is, of course, directly quasi-minimal. The following lemma gives an example of an uncountable decomposable torsion-free reduced directly quasi-minimal group which is not purely quasi-minimal.

Lemma 4.4. The Baer-Specker group $\prod_{\aleph_{0}} \mathbb{Z}$ is directly quasi-minimal but not purely quasi-minimal.

Proof. Let $P=\prod_{\aleph_{0}} \mathbb{Z}$. First note that $P$ is not purely quasi-minimal since it contains a pure free subgroup of rank $2^{\aleph_{0}}$ (see [5, Corollary 97.4]). If $P=A \oplus B$, then both $A$ and $B$ are products of countably many copies of $\mathbb{Z}$ (see [3, IX, Theorem 1.4]) and either $|A|=|P|=2^{\aleph_{0}}$ or $|B|=|P|=2^{\aleph_{0}}$ or both. Suppose that $A=\prod_{I} \mathbb{Z}$ and 
$|A|=|P|$. Since $|I|$ is countable and $|A|=|P|$ we must have $|I|=\aleph_{0}$ and hence $A \cong P$.

However, if we consider $G=\prod \mathbb{Z}$ where $\kappa>\aleph_{0}$, then the direct quasi-minimality of $G$ may be undecidable in $\mathrm{ZFC}$, as is shown by our final proposition.

Proposition 4.5. Let $\kappa>\aleph_{0}, \kappa$ not $\omega$-measurable, and let $G=\prod_{\kappa} \mathbb{Z}$. Then:

(i) assuming GCH, $G$ is directly quasi-minimal;

(ii) assuming $\mathrm{MA}+\neg \mathrm{CH}$, then, for all $\aleph_{0}<\kappa<2^{\aleph_{0}}, G$ is not directly quasiminimal.

Proof. (i) Suppose $G=A \oplus B$ with $|A|=|G|=2^{\kappa}$, say. Then $A=\prod_{I} \mathbb{Z}$ with $|I|=\lambda$, for some $\lambda \leq \kappa$, (see [3] IX, Theorem 1.4]) and so $2^{\lambda}=2^{\kappa}$. Assuming GCH we get $\lambda=\kappa$ and so $A \cong G$ and hence $G$ is directly quasi-minimal.

(ii) Now assume that MA $\rightarrow \mathrm{CH}$ holds. Let $G=\prod_{\kappa} \mathbb{Z}$ where $\aleph_{0}<\kappa<2^{\aleph_{0}}$, and we can assume that $2^{\aleph_{0}}=2^{\kappa}$, since this is a consequence of MA $+\neg \mathrm{CH}$ (see 3, p. 177]). Then $G=\prod_{\aleph_{0}} \mathbb{Z} \oplus B$, say, with $\left|\prod_{\aleph_{0}} \mathbb{Z}\right|=|G|$ but $\prod_{\aleph_{0}} \mathbb{Z} ¥ G$, since $\bigoplus_{\aleph_{0}} \mathbb{Z}=\left(\prod_{\aleph_{0}} \mathbb{Z}\right)^{*} \neq G^{*} \cong \bigoplus_{\kappa} \mathbb{Z}$ (see [3, III, Corollary 3.7]) and so $G$ is not directly quasi-minimal.

Since both GCH and MA+ $\neg \mathrm{CH}$ can be shown to be consistent with ZFC (see 14]) we can deduce that the direct quasi-minimality of e.g. $G=\prod_{\aleph_{1}} \mathbb{Z}$ is not decidable in ZFC. We note that the full strength of $\mathrm{MA}+\neg \mathrm{CH}$ is not required: we simply need any model consistent with ZFC in which $2^{\kappa}=2^{\aleph_{0}}$ holds for cardinals $\aleph_{0}<\kappa<2^{\aleph_{0}}$. Such a model may be obtained from any model of ZFC in which $2^{\aleph_{0}}$ is regular, by using Easton forcing with a constant index function equal to $2^{\aleph_{0}}$. Details of Easton forcing may be found in Kunen's book [10].

\section{REFERENCES}

1. A.L.S. Corner, Every countable reduced torsion-free ring is an endomorphism ring, Proc. London Math. Soc. 13 (1963) 687-710. MR 27:3704

2. A.L.S. Corner, On a conjecture of Pierce concerning direct decompositions of abelian groups, Proc. Colloq. Abelian Groups, Budapest (1964), 43-48. MR 30:148

3. P. C. Eklof and A. H. Mekler, Almost Free Modules, North-Holland Mathematical Library 46 (1990). MR 92e:20001

4. L. Fuchs, Infinite Abelian groups I, Academic Press, New York (1970). MR 41:333

5. L. Fuchs, Infinite Abelian groups II, Academic Press, New York (1973). MR 50:2362

6. J. Ginsburg and B. Sands, Minimal infinite topological spaces, Amer. Math. Monthly 86 (1979), 574-576. MR 80e:54007

7. P. Griffith, Infinite Abelian Groups, Chicago Lectures in Mathematics, Chicago and London (1970).

8. M. Holz, K. Steffens, and E. Weitz, Introduction to Cardinal Arithmetic, Birkhäuser Advanced Texts, Birkhäuser-Verlag, Basel-Boston-Berlin (1999). MR 2000h:03001

9. T. Jech, Set Theory, Academic Press (1978). MR 80a:03062

10. K. Kunen, Set Theory, North-Holland (1980). MR 82f:03001

11. J. Matier and T.B.M. McMaster, Iteration of the "anti" operation in ordered topological spaces and in other categorical contexts, Boll. Un. Mat. Ital. 7 (1993), 369-378. MR 94g:18002

12. P. T. Matthews and T.B.M. McMaster, A viewpoint on minimality in topology, Irish Math. Soc. Bull. 32 (1994), 7-16. 
13. S. Shelah, Infinite abelian groups, Whitehead problem and some constructions, Israel J. Math. 18 (1974), 243-256. MR 50:9582

14. R. M. Solovay and S. Tennenbaum, Iterated Cohen extensions and Souslin's problem, Ann. of Math. 94 (1971), 201-245. MR 45:3212

School of Mathematical Sciences, Dublin Institute of Technology, Kevin Street, Dublin 8, IRELAND

E-mail address: brendan.goldsmith@dit.ie

School of Mathematical Sciences, Dublin Institute of Technology, Kevin Street, Dublin 8, IRELAND

E-mail address: johog@maths.tcd.ie

Fachbereich 6, Mathematik und Informatik, Universität Essen, 45117 Essen, Germany

E-mail address: simone.wallutis@uni-essen.de 